\title{
Electric winds driven by time oscillating corona discharges
}

\section{Citation}

Drews, Aaron M., Ludovico Cademartiri, George M. Whitesides, and Kyle J. M. Bishop. 2013. "Electric Winds Driven by Time Oscillating Corona Discharges." Journal of Applied Physics 114(14): 143302.

\section{Published Version}

doi:10.1063/1.4824748

\section{Permanent link}

http://nrs.harvard.edu/urn-3:HUL.InstRepos:12361261

\section{Terms of Use}

This article was downloaded from Harvard University's DASH repository, and is made available under the terms and conditions applicable to Open Access Policy Articles, as set forth at http:// nrs.harvard.edu/urn-3:HUL.InstRepos:dash.current.terms-of-use\#OAP

\section{Share Your Story}

The Harvard community has made this article openly available.

Please share how this access benefits you. Submit a story.

Accessibility 


\title{
Electric winds driven by ac corona discharges
}

\author{
Aaron M. Drews, ${ }^{1}$ Ludovico Cademartiri ${ }^{2,3,4,5}$, George M. Whitesides ${ }^{2,6}$, \\ and Kyle J.M. Bishop ${ }^{1 a)}$ \\ ${ }^{1}$ Department of Chemical Engineering, Pennsylvania State University, University Park, PA, 16802, USA \\ ${ }^{2}$ Department of Chemistry \& Chemical Biology, Harvard University, Cambridge, MA, 02138 USA \\ ${ }^{3}$ Department of Materials Science \& Engineering, Iowa State University, Ames, IA, 50011, USA \\ ${ }^{4}$ Department of Chemical \& Biological Engineering, Iowa State University, Ames, IA, 50011, USA \\ ${ }^{5}$ Ames Laboratory, US Department of Energy, Ames, IA, 50011, USA \\ ${ }^{6}$ Kavli Institute, Harvard University, Cambridge, MA, 02138, USA
}

We investigate the formation of steady gas flows - so-called electric winds - created by point-plane corona discharges driven by time oscillating (ac) electric fields. By varying the magnitude and frequency of the applied field, we identify two distinct scaling regimes: (i) a low frequency (dc) regime and (ii) a high frequency (ac) regime. These experimental observations are reproduced and explained by a theoretical model describing the transport and recombination of ions surrounding the discharge and their contribution to the measured wind velocity. The two regimes differ in the spatial distribution of ions and in the process by which ions are consumed. Interestingly, we find that ac corona discharges generate strong electric forces localized near the tip of the point electrode, while dc corona discharges generate weaker forces distributed throughout the interelectrode region. Consequently, the velocity of the electric winds $(>1 \mathrm{~m} / \mathrm{s})$ generated by ac discharges is largely independent of the position of the counter electrode.

\footnotetext{
${ }^{a}$ Author to whom correspondence should be addressed. Electronic mail: kjmbishop@engr.psu.edu
} 
The unified theoretical description of dc and ac electric winds presented here reconciles previous observations of winds driven by dc corona and ac dielectric barrier discharges; insights from the model should also prove useful in the design of other plasma actuators.

\section{INTRODUCTION}

Gas flows driven by electric fields - so called ionic, electric, or corona winds - are achieved when ions generated by a corona discharge are accelerated by an applied field and transfer momentum to the surrounding gas. Unlike conventional forms of airflow control (e.g., fans and blowers) which require large moving parts and are often slow and noisy, electric gas actuators have fast response times, small size, and no moving parts. Consequently, electric winds can address problems that challenge conventional forms of gas actuation: controlling boundary layer dynamics in aeronautics ${ }^{1-3}$, improving heat transfer in confined spaces ${ }^{4-6}$, modifying surface properties ${ }^{7,8}$, and removing atmospheric contaminants ${ }^{9-11}$.

There are two common designs for electric wind actuators: 1) "point-plane" actuators ${ }^{12}$, and 2) dielectric barrier discharge (DBD) actuators ${ }^{13-21}$. In the former, a static voltage (typically several to tens of $\mathrm{kV}$ ) is applied between an electrode of high curvature - the "point", usually a needle or wire - and a low curvature counter electrode - the "plane", often a plate or ring positioned some distance away (Fig. 1(a)). A localized ionization region - that is, a corona discharge $\mathrm{e}^{12,22}$ - forms at the point electrode and produces charged species (for air, primarily $\mathrm{CO}^{3-}, \mathrm{O}^{3-}, \mathrm{HCO}^{3-}$ and $\mathrm{NO}^{3-}$ from negative corona ${ }^{23}$ and $\mathrm{H}_{3} \mathrm{O}^{+}, \mathrm{NO}^{+}$, and $\mathrm{NO}^{2+}$ from positive corona $^{24}$ ) that drift in the field toward the counter electrode. Electric wind is produced by collisional momentum transfer from these ions to the surrounding neutral species. The relatively 
simple point-plane geometry is well suited for experimental characterization - e.g., the parametric optimization of wind velocity - and has been studied both experimentally ${ }^{25-29}$ and theoretically. ${ }^{25,30-32}$ Because the electric force driving the flow depends on the field between the electrodes, maximum flow rates are achieved for closely spaced electrodes and fall precipitously as electrode separation increases ${ }^{27,33}$ ( $c f$. below). The use of static electric fields further requires a steady current of ions which must migrate unobstructed from one electrode to the other.

By contrast, DBD actuators are characterized by the presence of a dielectric slab (e.g., PMMA $^{13}$, glass ${ }^{14}$ or glass-filled epoxy ${ }^{15}$ ) positioned between two high curvature electrodes (Fig. 1(a)). Here, a static field is ineffective: charged species generated within the corona rapidly accumulate on the dielectric surface, which screens the applied field and limits the ion current and the resulting gas flow to near zero ${ }^{16}$. Instead, time-varying voltages - often harmonic with frequencies of $10^{2}-10^{3} \mathrm{~Hz}^{17}$ - are applied across the electrodes to produce a variety of electric breakdown phenomena ${ }^{22}-$ e.g., corona, streamers, and plasma, though spark breakdown is prevented by the dielectric barrier. Somewhat counter-intuitively, the transient migration of charged species within ac fields also gives rise to steady electric winds. ${ }^{14,15,18,19}$ Simulation studies $^{20,} 21$ have shown how the processes of ionization, ion/electron transport, and recombination result in a time-averaged, electrohydrodynamic force that drives the gas flows observed in experiments.

Here, we attempt to unify the two distinct mechanisms of dc and ac electric winds through experimental and scaling analysis of a simple model system - the point-ring electrode geometry shown in Fig. 1(b). As we show, this system is capable of generating electric winds (with 
velocities of few $\mathrm{m} / \mathrm{s}$ ) for both static (dc) and time-varying (ac) applied voltages. By quantifying the wind velocity as a function of frequency, voltage, and electrode separation, we identify two distinct scaling regimes - a low frequency $(\mathrm{dc})$ regime $^{27}$ and a high frequency (ac) regime. The observed scaling trends are reproduced by a theoretical model ${ }^{34}$, which explains the physical mechanisms underlying both $\mathrm{dc}$ and ac winds. In the ac regime, ions generated within the corona move in the field and migrate a distance $\delta$ ( $c f$. below) before recombining; the net flow of ions away from the corona creates a time-averaged force that drives the steady flows observed experimentally. Importantly, because the electric force is localized near the point electrode, ac coronas can sustain wind velocities of $>1 \mathrm{~m} / \mathrm{s}$ independent of electrode separation - in marked contrast to de coronas. Building on insights from model and experiment, we investigate how multiple ac coronas can be "stacked" in parallel to enhance the overall wind velocity.

\section{EXPERIMENT}

The experimental setup is illustrated in Fig. 1(b). A stainless steel needle (DMC 1767/6, tip radius $\sim 70 \mu \mathrm{m}$ ) was centered coaxially within a quartz tube (inner diameter $D=2.2 \mathrm{~cm}$ ) with its tip a distance $L$ from a cylindrical aluminum counter-electrode. Time-varying (sinusoidal) electric potentials were applied to the needle using a high voltage amplifier (Trek 40/15) driven by a function generator (Keithley 3390) with a sinusoidal waveform $V=\sqrt{2} V_{0} \cos (\omega t)$ where the root-mean-square (rms) voltage $V_{0}$ was varied from 5-28 $\mathrm{kV}$ and the frequency $v=\omega / 2 \pi$ from $1-10^{3} \mathrm{~Hz}$. To characterize the ionic wind, we measured the outlet velocity $u_{o}$ using a hot wire anemometer (TSI 8455, Fig. 1(b)). As noted for DBD actuators, ${ }^{13,35}$ the ac waveform produces a pulsed wind oscillating at twice the frequency of the driving signal; with a sampling period of $0.25 \mathrm{~s}$, the anemometer provided a time-averaged velocity measurement. To focus the gas flow 
onto the anemometer, a quartz nozzle was placed after the counter-electrode (outlet diameter, $D_{\mathrm{o}}$ $=1.0 \mathrm{~cm}$ ); the entire structure was enclosed in a transparent box to mitigate the effects of air movement in the surrounding environment. For studies with multiple point electrodes, a polyethylene tube (12.2 cm inner diameter) was used in place of the quartz tube; a strip of aluminum adhesive tape on the inside of the tube served as a counter electrode.

\section{RESULTS}

Fig. 1(c) shows the electric wind velocity $u_{o}$ as a function of the electrode separation $L$ for several frequencies $(v=1-2000 \mathrm{~Hz})$ and a constant applied voltage of $V_{0}=12 \mathrm{kV}_{\text {rms. At low }}$ frequencies $(v \sim 1 \mathrm{~Hz})$, the wind velocity $u_{o}$ decreased with increasing electrode separation $L$ as captured by the approximate scaling relation, $u_{o} \propto L^{-1}$. Winds driven by positive or negative $\mathrm{dc}$ potentials exhibited a similar behavior; however, the accumulation of charged species on the walls of the tube prohibited a quantitative comparison when the electrodes were separated by more than $10 \mathrm{~cm}$ (see Supporting Information, Section 3). In contrast, the wind velocity at high frequencies $(v>500 \mathrm{~Hz})$ was largely independent of the electrode separation, $u_{o} \propto L^{0}$. The wind velocity at intermediate frequencies transitioned smoothly between these limiting regimes, which suggests that common mechanisms underlie the formation of dc (low frequency) and ac (high frequency) electric winds ( $c f$. below).

The frequency dependent transition in the wind velocity is shown more clearly in Fig. 2(a), which plots the wind velocity $u_{o}$ as a function of frequency $v$ for several applied voltages $\left(V_{0}=\right.$ $\left.8-16 \mathrm{kV}_{\mathrm{rms}}\right)$ and a constant electrode separation of $L=10 \mathrm{~cm}$. Wind velocity increased with increasing frequency approaching a constant value above some critical frequency $(\sim 500 \mathrm{~Hz})$. 
Regardless of frequency, the wind velocity increased linearly with the applied voltage, $u_{o} \propto V_{0}$ (Fig. 2(b)). Deviations from this linear scaling were observed only at low voltages $\left(V_{0}<8\right.$ $\mathrm{kV}_{\mathrm{rms}}$ ) near the corona inception voltage, below which no corona (and no wind) was generated ${ }^{12}$.

\section{DISCUSSION}

To explain these observations, we consider the field-induced transport of charged species generated within the corona and their effect on the neutral gas. In general, electric winds are driven by an electric force $\mathbf{f}_{e}(\mathbf{x}, t)=\rho_{e} \mathbf{E}$, which enters the Navier-Stokes equations governing fluid motion (here, $\rho_{e}$ is the charge density due to mobile ions/electrons, and $\mathbf{E}$ is the local electric field). As we have shown ${ }^{34}$, even for ac fields, the time-averaged electric force is nonzero and directed outward from the corona discharge. This steady component of the electric force drives the gas flows observed in experiment.

Importantly, the spatial distribution of the steady force depends of the frequency of the applied field. At low frequencies (here, $<100 \mathrm{~Hz}$ ), the distribution of charged species - and thereby the electric force - extends throughout the inter-electrode region (Fig. 3(a)). At high frequencies (here, $>1000 \mathrm{~Hz}$ ), the charged species and the electric force become increasingly localized near the tip of the point electrode (Fig. 3(c)). Below we use a simplified model of charge transport to estimate the total electric force driving the gas flows in both regimes. The estimates of the electric force allow us to compare the model's predictions to the observed gas velocity.

\section{A. Hydrodynamics}


To relate the electric force $F_{e}$ to the gas velocity $u_{o}$, we consider a macroscopic control volume enclosing the gas within the quartz tube. Conservation of mass implies that $D^{2} u \approx D_{o}^{2} u_{o}$ where $u_{o}$ is the average gas velocity through the outlet nozzle (the experimental observable), and $u$ is the velocity within the tube. Additionally, conservation of momentum implies that

$$
\rho u_{o}^{2} D_{o}^{2}-\rho u^{2} D^{2} \approx F_{e}-F_{v i s c}
$$

where $F_{e}$ is the electric force (acting in the direction of flow), $F_{v i s c} \sim \mu u L$ is the total viscous force resisting the flow, and $\rho$ and $\mu$ are the density and viscosity of air. Assuming that $D^{2} / D_{o}^{2}>>1$ and $\rho u_{o} D^{2} / \mu L>>1$ as in experiment $\left(D^{2} / D_{o}^{2} \approx 5\right.$ and $\left.\rho u_{o} D^{2} / \mu L \approx 100\right)$, the dominant terms of Eq. (1) suggest that $F_{e} \sim \rho u_{o}^{2} D_{o}^{2}$. The measured wind velocity should therefore scale with the electric force as

$$
u_{o} \sim \sqrt{\frac{F_{e}}{\rho D_{o}^{2}}}
$$

This scaling result is further supported by additional experiments using a small gas jet to mimic the localized electric force $F_{e}$ (see Supporting Information, Section 4).

\section{B. Charge Transport}

To estimate the electric force, we consider a simplified charge transport model ${ }^{34}$ that describes (1) the field induced migration of ions within the inter-electrode region, (2) their influence on the applied field, and (3) their consumption by recombination processes. ${ }^{16,34}$ The number densities $n_{ \pm}(\mathbf{x}, t)$ of positive and negative ions are governed by the conservation equation

$$
\frac{\partial n_{ \pm}}{\partial t}+\nabla \cdot\left( \pm K n_{ \pm} \mathbf{E}\right)=-k_{r} n_{+} n_{-}
$$


Here, the charge carriers are assumed to be monovalent ions with equal mobilities $\pm K \sim 10^{-4} \mathrm{~m}^{2} / \mathrm{V} \cdot \mathrm{s} ; k_{r} \sim 10^{-13} \mathrm{~m}^{3} /$ ions $\cdot \mathrm{s}^{36}$ is the rate constant for ion-ion recombination; and the electric field $\mathbf{E}(\mathbf{x}, t)$ is governed by Gauss's law:

$$
\nabla \cdot \mathbf{E}=\frac{e}{\varepsilon_{0}}\left(n_{+}-n_{-}\right)
$$

At the tip of the point electrode, we assume that the corona discharge maintains a constant ion density, $n_{ \pm}=n_{o} \sim 10^{17}$ ions $/ \mathrm{m}^{3} 37,38$; the counter electrode is assumed to be perfectly absorbing, $n_{ \pm}=0$. By treating the corona as a boundary condition, we avoid the complications of modeling the discharge itself. As we will show, the electric force driving the gas flow is distributed throughout the inter-electrode region - not within the corona. For this reason, we also neglect the effects of electrons, which rapidly attach to $\mathrm{O}_{2}$ in air to form negative ions (on time scales of $\sim 10 \mathrm{~ns}^{39}$ ). Other transport mechanisms - namely, convection and diffusion - were negligible compared to the field induced drift. The typical drift velocity is $K E \sim 10-100 \mathrm{~m} / \mathrm{s}$ as compared to that of convection $u \sim 1 \mathrm{~m} / \mathrm{s}$ and diffusion $D_{i} / a \sim 0.1 \mathrm{~m} / \mathrm{s}$, where $D_{i} \sim 10^{-5} \mathrm{~m}^{2} / \mathrm{s}$ is the ion diffusion coefficient, and $a \sim 100 \mu \mathrm{m}$ is the radius of curvature of the point electrode.

1. dc Limit. For static fields, only ions of one polarity are present outside of the corona, and they extend throughout the inter-electrode region (Fig. 3(a)). In this limit, we can approximate the point-plane geometry by a 1-dimensional Cartesian coordinate system ${ }^{27}$. With these assumptions, Eqs. (3) and (4) simplify in the case of a positive corona to give

$$
\frac{d}{d x}\left(K n_{+} E\right)=0 \text { and } \frac{d E}{d x}=\frac{e}{\varepsilon_{0}} n_{+}
$$

While these equations can be solved analytically (see Supporting Information, Section 1), we limit our discussion to order of magnitude estimates. Approximating the electric field as 
$E \sim V_{0} / L$ and spatial derivatives as $d / d x \sim L^{-1}$, Eq. (5) implies that $n_{+} \sim \varepsilon_{0} V_{0} / e L^{2}-$ the ion density in the inter-electrode region is proportional to the applied field. The electric force density can therefore be approximated as

$$
f_{e} \sim e n_{+} E \sim \frac{\varepsilon_{0} V_{0}^{2}}{L^{3}}
$$

Integrating over the entire inter-electrode region (i.e., the cylinder of length $L$ and diameter $D$ ), the total electric force can be approximated as

$$
F_{e} \sim D^{2} L f \sim \frac{D^{2}}{L^{2}} \varepsilon_{0} V_{0}^{2}
$$

Using Eq. (2), the gas velocity scales as

$$
u_{o} \sim \sqrt{\frac{\varepsilon_{0}}{\rho}} \frac{D}{D_{o}} \frac{V_{0}}{L}
$$

The wind velocity increases linearly with the applied voltage and is inversely proportional to the electrode separation. These predicted trends are identical to those observed in experiments in the low frequency limit (Fig. 1(c) and Fig. 2). Furthermore, substituting the experimental parameters $\left(V_{0} \sim 10 \mathrm{kV}, L \sim 10 \mathrm{~cm}\right)$ the wind velocity is estimated to be $u_{o} \sim 0.6 \mathrm{~m} / \mathrm{s}$ in qualitative agreement with experiment.

From a microscopic perspective, ions generated in the corona travel a characteristic distance $L$ to the counter electrode where they are consumed. Each ion creates an impulse (force multiplied by time) of order $e L / K$, which is transferred to the neutral gas through molecular collisions. Multiplying this impulse by the net flux of ions leaving the ionization region yields the electric force $F_{e}$ driving the flow. Using the scaling arguments above, the total ion flux (ions 
reaching the counter-electrode per unit time) may be approximated as $D^{2} K n_{+} E \sim D^{2} K \varepsilon_{0} V_{0}^{2} / e L^{3}$ such that $F_{e} \sim D^{2} \varepsilon_{0} V_{0}^{2} / L^{2}$ as derived in Eq. (7).

2. ac Limit. For sufficiently high frequencies (to be determined), ions generated in the corona no longer reach the absorbing counter-electrode but are rather consumed by ion-ion recombination processes within the inter-electrode region (Fig. 3(b) and (c)). Consequently, the ion distribution extends only a finite distance $\delta_{x}<L$, which decreases with increasing frequency. To determine the length scale $\delta_{x}$ and its dependence on frequency, we rewrite Eqs. (3) and (4) in terms of the total ion density $n_{s}=n_{+}+n_{-}$and the charge density $n_{d}=n_{+}-n_{-}$, retaining only the dominant terms.

$$
\frac{\partial n_{s} / s}{\partial t}+\frac{\partial}{\partial x}\left(K n_{d} E\right)=-\frac{1}{2} k_{r}\left(n_{s}^{2}-\not y_{d}^{\not}\right), \frac{\partial n_{d}}{\partial t}+\frac{\partial}{\partial x}\left(K n_{s} E\right)=0, \text { and } \frac{\partial E}{\partial x}=\frac{e}{\varepsilon_{o}} n_{d}
$$

As with the de limit above, we've approximated the point plane geometry by a 1-dimensional Cartesian coordinate system, for which the electric field scales as $E \sim V_{0} / L$. Approximating time derivatives as $\partial / \partial t \sim \omega$ and spatial derivatives as $d / d x \sim \delta_{x}^{-1}$, Eq. (9) implies that

$$
\frac{K n_{d} V_{o}}{\delta_{x} L} \sim k_{r} n_{s}^{2}, \omega n_{d} \sim \frac{K n_{s} V_{o}}{\delta_{x} L} \text {, and } \frac{V_{o}}{\delta_{x} L} \sim \frac{e n_{d}}{\varepsilon_{o}}
$$

Solving for the unknown scales $-n_{d}, n_{s}$, and $\delta_{x}-$ we find that $n_{s} \sim \varepsilon_{0} \omega / e K, n_{d} \sim \kappa \varepsilon_{0} \omega / e K$, and $\delta_{x} \sim K V_{o} / \kappa L \omega$, where $\kappa^{2}=\varepsilon_{0} k_{r} / e K \sim 0.1$ is a dimensionless parameter characterizing the rates of ion recombination and ion screening. Examining the magnitude of the neglected terms, this dominant balance is appropriate provided $\delta_{x}=L$ and $\kappa=1$ (see Supporting Information, Section 1 for a more thorough analysis of Eq. (9)). The frequency dependent transition from the 
dc limit to ac limit is expected to occur when $\delta_{x} \sim L$, which corresponds to a crossover frequency of $\omega^{*} \sim K V_{o} / \kappa L^{2}$. For the experimental parameters $\left(K \sim 10^{-4} \mathrm{~m}^{2} / \mathrm{V} \cdot \mathrm{s}, V_{0} \sim 10 \mathrm{kV}_{\mathrm{rms}}\right.$, $\kappa^{2} \sim 0.1$, and $L \sim 10 \mathrm{~cm}$ ), this frequency is estimated to be $v^{*}=\omega^{*} / 2 \pi \sim 50 \mathrm{~Hz}$.

With these scaling estimates, the resulting force density is approximated as

$$
f_{e} \sim e n_{d} E \sim \frac{\kappa \varepsilon_{0} \omega V_{o}}{K L} \sim \frac{\varepsilon_{0} V_{o}^{2}}{\delta_{x} L^{2}}
$$

Integrating over the entire ion distribution (i.e., the cylinder of length $\delta_{x}$ and diameter $D$ ), the total electric force can be approximated as

$$
F_{e} \sim \delta_{x} D^{2} f \sim \frac{D^{2}}{L^{2}} \varepsilon_{0} V_{o}^{2}
$$

This result is identical to that derived for de fields and yields the same wind velocity (Eq. (7)). From the microscopic perspective, each ion migrates a shorter distance $\delta_{x}<L$ on average before recombining with an ion of opposite charge; consequently, the impulse created by each ion is reduced from $e L / K$ in the dc limit to $e \delta_{x} / K$ in the ac limit. However, this reduction in the migration distance is exactly balanced by an increase in the ion flux, which is now $D^{2} K n_{d} E \sim D^{2} K \varepsilon_{0} V_{o}^{2} / e \delta_{x} L^{2}$

In sum, the transition from the dc limit, where ions are consumed at the counter electrode, to the ac limit, where ions are consumed by ion recombination, is not sufficient to explain the increase in the electric wind velocity observed in experiment. The failure of the above description to capture the experimental results derives from the use of the 1-dimensional Cartesian coordinate system to describe the point-plane electrode geometry. At high frequencies, 
the ion distribution is increasingly localized near the tip of the point electrode; consequently, we must account for the curvature of the electrode to accurately capture the ac regime observed in experiment.

3. Curvature Effects in the ac Limit. To approximate the electrode geometry near the tip of the point electrode, we again consider Eqs. (3) and (4) using a 1-dimensional, spherical coordinate system.

$$
\frac{1}{r^{2}} \frac{\partial}{\partial r}\left(r^{2} K n_{d} E\right) \approx-\frac{1}{2} k_{r} n_{s}^{2}, \frac{\partial n_{d}}{\partial t}+\frac{1}{r^{2}} \frac{\partial}{\partial r}\left(r^{2} K n_{s} E\right)=0, \frac{1}{r^{2}} \frac{\partial}{\partial r}\left(r^{2} E\right)=\frac{e}{\varepsilon_{o}} n_{d}
$$

In this geometry, the characteristic electric field depends not on the length scale $L$ but rather on the size of the (spherical) ion distribution $\delta_{r}$ as $E \sim V_{0} / \delta_{r}$. Approximating time derivatives as $\partial / \partial t \sim \omega$ and spatial derivatives as $d / d r \sim \delta_{r}^{-1}$, Eq. (13) implies that the length scale $\delta_{r}$ may be approximated as $\delta_{r} \sim\left(K V_{0} / \kappa \omega\right)^{1 / 2}$. With this correction, the force density is given by

$$
f_{e} \sim e n_{d} E \sim \frac{\kappa \varepsilon_{0} \omega V_{0}}{K \delta_{r}}
$$

Integrating over the volume of the ion distribution, the total force is approximated as

$$
F_{e} \sim \delta_{r}^{3} f \sim \varepsilon_{0} V_{0}^{2}
$$

This is considerably larger than the de limit of Eq. (7) by a factor of $L^{2} / D^{2} \sim 25$. The resulting gas velocity is

$$
u_{o} \sim \sqrt{\frac{\varepsilon_{0}}{\rho}} \frac{V_{0}}{D_{o}}
$$


Importantly, this velocity is independent of the electrode separation $L$ and scales linearly with the applied voltage $V_{0}$ as observed in experiment. Furthermore, the wind velocity is larger than that in the de limit by a factor of $L / D \sim 5$.

The transition from the dc limit to ac limit should occur when the length scale $\delta_{r} \sim\left(K V_{0} / \kappa \omega\right)^{1 / 2}$ is comparable to the diameter of the tube, which corresponds to a crossover frequency of $\omega^{\dagger} \sim K V_{o} / \kappa D^{2}$. For the experimental parameters, this frequency is estimated to be $v^{\dagger} \sim 1000 \mathrm{~Hz}$. In experiment, this corresponds to the onset of the plateau region.

\section{Multiple Electrodes}

Even in the ac regime $(v>1000 \mathrm{~Hz})$, the electric wind velocity is limited to few meters per second by electrical breakdown in $\operatorname{air}^{27}$ (when $V_{0} / \delta_{r}>10^{6} \mathrm{~V} / \mathrm{m}$ ) and by the limits of the high voltage amplifier. Previous efforts to increase the electric wind velocity have relied on multiple electrodes arranged series using either point-plane ${ }^{27}$ or $\mathrm{DBD}^{35,40,41}$ actuators. Here, we investigate the possibility of enhancing the wind velocity using multiple point electrodes arranged in parallel. At high frequencies $(v>1000 \mathrm{~Hz})$, the electric force is localized near the tip of the point electrode within a region of size $\delta_{r}<2 \mathrm{~cm}$. By incorporating multiple electrodes separated by a distance $d$ larger than $\delta_{r}$, each corona should contribute independently to the total electric force $F_{e}$ thereby increasing the measured wind velocity.

To evaluate this hypothesis, we positioned three point electrodes in parallel at the vertices of an equilateral triangle (side length, $d=6.6 \mathrm{~cm})$ inside a large tube $(D=12.2 \mathrm{~cm})$, applied an ac potential $\left(v=1000 \mathrm{~Hz}, V_{0}=10 \mathrm{kV}\right)$, and measured the wind velocity $u_{o}$ at the outlet nozzle 
$\left(D_{o}=2 \mathrm{~cm}\right)$ (Fig. 4(a)). Rather than change the number of electrodes, we varied the number of active corona $N$ by capping some of the point electrodes in silicone; in this way, we maintained the same applied field and the same hydrodynamic obstacles for each value of $N$. Surprisingly, there was no significant enhancement of the wind velocity for $N=2$ or 3 coronas as compared to that of a single corona (Fig. 4(b)).

To understand this result, we note that the size of the ion distribution scales as $\delta_{r} \sim K E / \kappa \omega$, where $E$ is the field at the surface of the ion cloud (a distance $\delta_{r}$ from the corona). Therefore, Eqs. (14) and (15) imply that the total electric force scales with the field as $F_{e} \sim e n_{d} E \delta_{r}^{3} \propto E^{4}$. Consequently, small changes to the electric field can have large effects on the electric force. Even when their ion clouds do not overlap, the presence of one corona acts to reduce the magnitude of the electric field at a neighboring corona thereby reducing the electric force $F_{e}$. In particular, for spherical ion clouds of size $\delta_{r}$ the characteristic field $E$ depends on the separation $d$ as $E \sim E_{\infty}\left(1-c \delta_{r} / d\right)$, where $E_{\infty} \sim V_{0} / \delta_{r}$ and $c$ is an order one constant (see Supporting Information, Section 2).

For small electrode separations $\left(d<d^{*}\right)$, the total force due to multiple corona, $N F_{N} \propto N E^{4}$, is actually predicted to be less than that of a single corona, $F_{1}(d) \propto E_{\infty}^{4}$. This prediction is confirmed experimentally as illustrated in Fig. 4(c), which shows that two closely spaced coronas $(d=1-10 \mathrm{~cm})$ produce wind velocities of $\sim 0.3 \mathrm{~m} / \mathrm{s}$ as compared to $\sim 0.5 \mathrm{~m} / \mathrm{s}$ for a single corona for otherwise identical conditions $\left(V_{0}=10 \mathrm{kV}, v=1.8 \mathrm{kHz}\right)$. At some critical separation $d^{*}=c \delta_{r} /\left(1-N^{-1 / 4}\right)$, the total force due to multiple coronas becomes comparable to 
that of a single corona - that is, $F_{1}=N F_{N}\left(d^{*}\right)$. For the experimental conditions used in Fig. 4(b), the electrode separation $d=6.6 \mathrm{~cm}$ is comparable to this critical length scale $d^{*} \sim 6 \mathrm{~cm}$, and there is only negligible enhancement of the wind velocity. Owing to the long range of electrostatic interactions, multiple electrodes would have to be separated by a distance $d>d^{*} \sim 6 \mathrm{~cm}$ for significant wind enhancement.

\section{CONCLUSIONS}

Experimental investigation of the electric wind velocity as a function of frequency, voltage, and electrode separation reveals two distinct scaling regimes - a low frequency (dc) regime and a high-frequency (ac) regime. These different scaling behaviors are explained by a theoretical model that describes the generation of ions within the corona, their migration in the field, and their consumption by recombination processes. In contrast to winds driven by static (dc) fields, ac fields generate wind velocities comparable to (or better than) the strongest dc winds for any value of the electrode separation $L$. In the high-frequency (ac) regime, the electric force is localized within a region of size $\delta_{r} \sim\left(K V_{0} / \kappa \omega\right)^{1 / 2}$ near the tip of the point electrode. The scaling results presented here should be useful in designing new types of electric wind actuators. From a fundamental perspective, we have shown how any type of electric wind (dc or ac) derives from the same basic mechanism whereby a steady flux of ions transfers momentum to the surrounding fluid to drive steady gas flows.

ACKNOWLEDGMENTS This work was supported by the Defense Advanced Research Projects Agency under Grant No. W944NF-09-1-0005, and by the Non-equilibrium Energy Research Center (NERC) - an Energy Frontier Research Center funded by the U.S. Department 
of Energy under Award Number DE-SC0000989. LC is grateful to Iowa State University for startup funding.

\section{REFERENCES}

1. T. C. Corke, C. L. Enloe and S. P. Wilkinson, Ann. Rev. Fluid Mech. 42, 505 (2010).

2. Post and Corke, "Separation control using plasma actuators: dynamic stall control on an oscillating airfoil," AIAA Paper No. 2004-2517, 2004.

3. Corke and Post, "Plasma flow control optimized airfoil," AIAA Paper No. 2006-1208, 2006.

4. D. B. Go, S. V. Garimella, T. S. Fisher and R. K. Mongia, J. Appl. Phys. 102, 053302 (2007).

5. M. Molki and K. L. Bhamidipati, Int. J. Heat Mass Trans. 47, 4301 (2004).

6. M. M. Ohadi, D. A. Nelson and S. Zia, Int. J. Heat Mass Trans. 34, 1175 (1991).

7. Z. Fang, Y. Qiu and Y. Luo, J. Phys. D-Appl. Phys. 36, 2980 (2003).

8. N. De Geyter, R. Morent, C. Leys, L. Gengembre and E. Payen, Surf. Coat. Technol. 201, 7066 (2007).

9. $\quad$ K. H. Yoo, J. S. Lee and M. D. Oh, Aero. Sci. Tech. 27, 308 (1997).

10. Y. Zhuang, Y. J. Kim, T. G. Lee and P. Biswas, J. Electrostatics 48, 245 (2000).

11. L. Li and D. R. Chen, J. Aerosol. Sci. 42, 87 (2011).

12. L. B. Loeb, Electrical Coronas. (University of California Press, Berkeley, CA, 1965).

13. M. Forte, L. Leger, J. Pons, E. Moreau and G. Touchard, J. Electrostatics 63, 929 (2005).

14. J. Pons, E. Moreau and G. Touchard, J. Phys. D-Appl. Phys. 38, 3635 (2005).

15. Roth, Sherman and Wilkinson, AIAA Journal 38, 1166 (2000). 
16. J. P. Boeuf and L. C. Pitchford, J. Appl. Phys. 97, 103307 (2005).

17. N. Benard and E. Moreau, App. Phys. Lett. 100, 193503 (2012).

18. Roth, Sherman and Wilkinson, "Boundary layer flow control with a one atmosphere uniform glow discharge surface plasma," AIAA Paper No. 1998-328, 1998.

19. C. L. Enloe, T. E. McLaughlin, R. D. Van Dyken, K. D. Kachner, E. J. Jumper and T. C. Corke, AIAA Journal 42, 589 (2004).

20. Y. Lagmich, T. Callegari, T. Unfer, L. C. Pitchford and J. P. Boeuf, App. Phys. Lett. 90, 051502 (2007).

21. J. P. Boeuf and et al., J. Phys. D-Appl. Phys. 40, 652 (2007).

22. J. M. Meek and J. D. Craggs, Electrical Breakdown of Gases. (John Wiley \& Sons, New York, NY, 1978).

23. J. D. Skalny, J. Orszagh, N. J. Mason, J. A. Rees, Y. Aranda-Gonzalvo and T. D. Whitmore, Int. J. Mass Spect. 272, 12 (2008).

24. M. M. Shahin, J. Chem. Phys. 45, 2600 (1966).

25. L. Zhao and K. Adamiak, J. Electrost. 63, 337 (2005).

26. M. Rickard, D. Dunn-Rankin, F. Weinberg and F. Carleton, J. Electrostatics 63, 711 (2005).

27. M. Rickard, D. Dunn-Rankin, F. Weinberg and F. Carleton, J. Electrostatics 64, 368 (2006).

28. S. Ohyama and R. Ohyama, in Annual Report Conference on Electrical Insulation and Dielectric Phenomena, Cancun, Mexico, 16-19 Oct. 2011, pp. 227-230.

29. E. Moreau and G. Touchard, J. Electrostatics 66, 39 (2008).

30. M. Rickard and D. Dunn-Rankin, J. Electrostatics 65, 646 (2007). 
31. E. A. Christensen and P. S. Moller, AIAA Journal 5, 1768 (1967).

32. L. Pekker and M. Young, J. Propul. Power 27, 786 (2011).

33. Y. Kitahara, K. Aoyagi and R. Ohyama, in 2007 Annual Report Conference on Electrical Insulation and Dielectric Phenomena, Vancouver, Canada, 14-17 Oct. 2007, pp. 529532.

34. A. M. Drews, L. Cademartiri, M. L. Chemama, M. P. Brenner, G. M. Whitesides and K. J. M. Bishop, Phys. Rev. E 86, 036314 (2012).

35. M. Forte, J. Jolibois, J. Pons, E. Moreau, G. Touchard and M. Cazalens, Exp. Fluids 43, 917 (2007).

36. A. B. Fialkov, Progress in Energy and Combustion Science 23, 399 (1997).

37. R. Morrow, Physical Review A 32 (1985).

38. R. Morrow, J. Phys. D-Appl. Phys. 30 (1997).

39. A. Dogariu, M. N. Shneider and R. B. Miles, in 2010 Conference on Lasers and ElectroOptics, San Jose, CA, 16-21 May 2010, pp. 1-2.

40. Borghi, Cristofolini, Carraro and Neretti, "An analysis of a three phase flat panel uniform barrier discharge at atmospheric pressure," AIAA Paper No. 2006-3380, 2006.

41. M. Riherd and S. Roy, J. Appl. Phys. 112, 053303 (2012). 
(a)

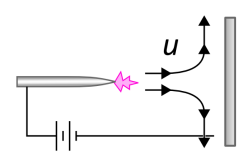

corona discharge

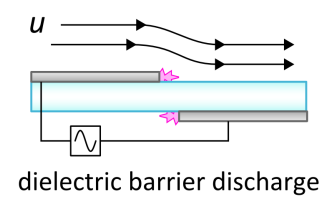

(b)

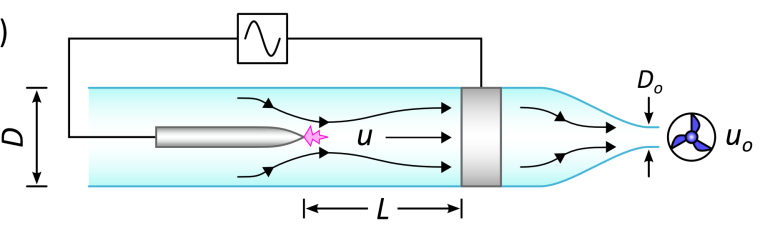

(c)

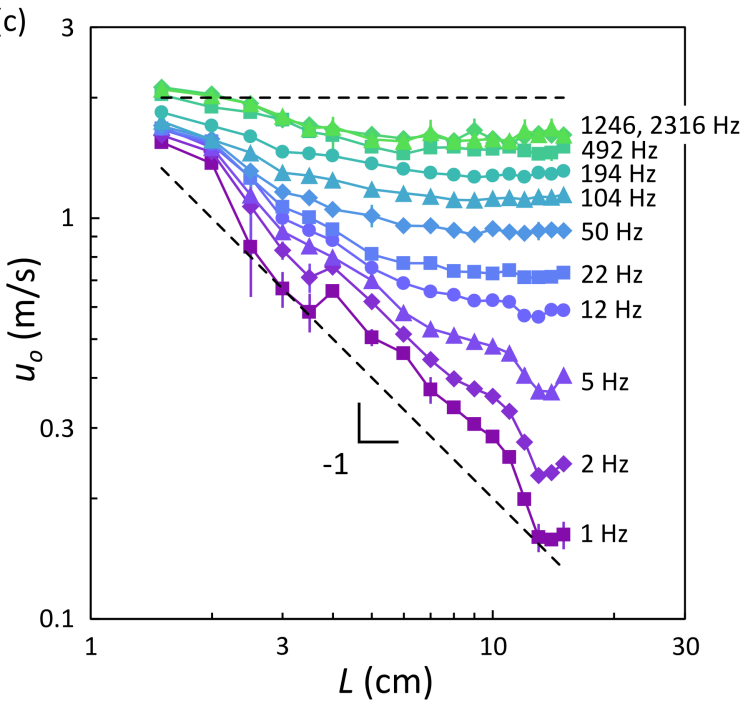

FIG. 1. Electric winds. (a) Schematic illustration of point-plane electric wind actuator (left) and DBD actuator (right). (b) Upon application of an electric potential (dc or ac) a corona discharge formed at the tip of a needle inside a quartz tube positioned a distance $L$ from an axially-aligned ring electrode; after $15 \mathrm{~s}$ the wind velocity $u_{o}$ at the tube outlet was measured using a hot wire anemometer over a period of 15 s. (c) Log-log plot of the wind velocity as a function of separation $L$ for ac winds $\left(V_{0}=12 \mathrm{kV}\right)$ at several frequencies. Error bars are standard deviations of five measurements; lines are to guide the eye. 

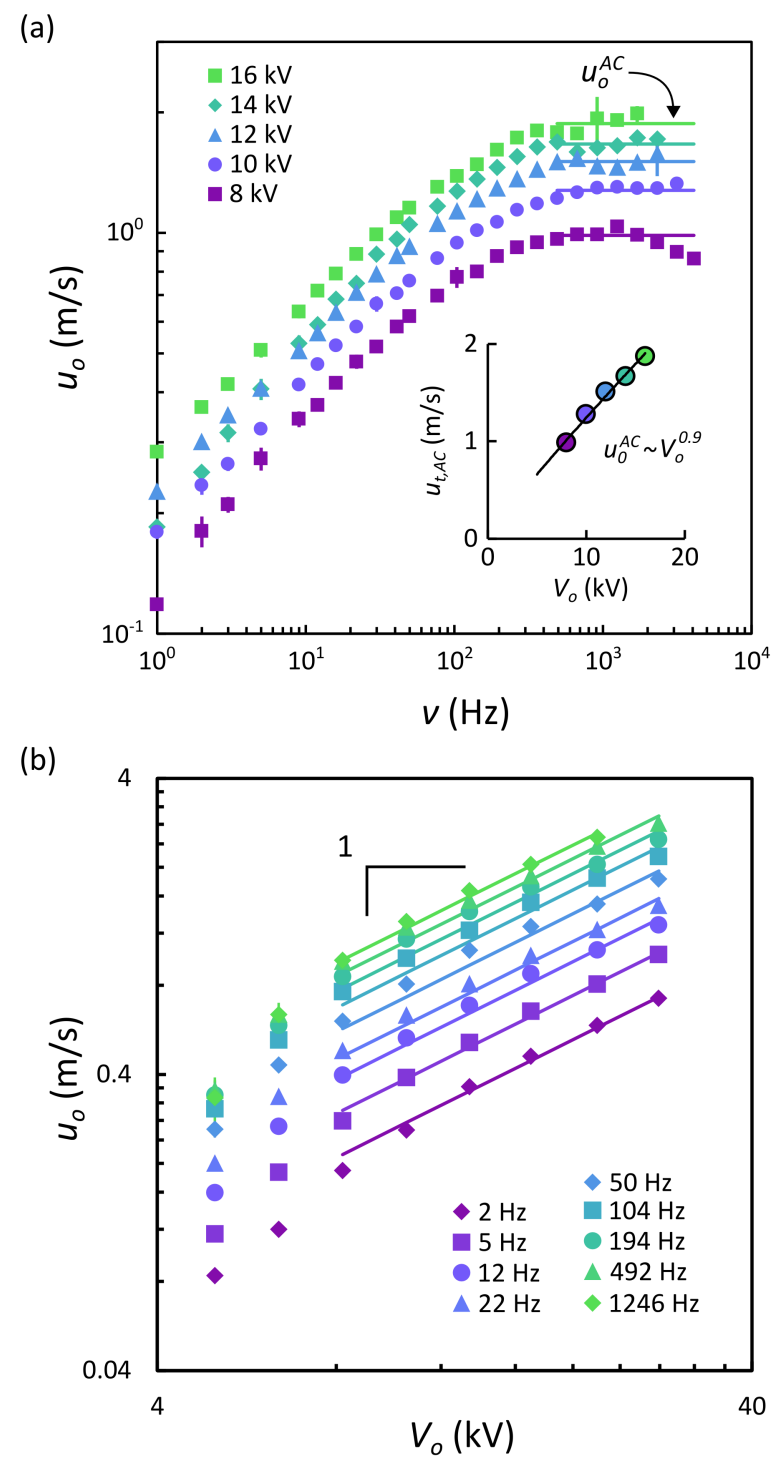

FIG. 2. Frequency and voltage dependence. (a) Log-log plot of the ac wind velocity $u_{o}$ as a function of frequency at several voltages for $L=10 \mathrm{~cm}$. Error bars are standard deviations; lines are means of all velocities above $500 \mathrm{~Hz}\left(u_{o}^{A C}\right)$ which scale nearly linearly with voltage (inset), in agreement with Eq. (16). (b) Log-log plot of the ac wind velocity $u_{o}$ as a function of voltage ( 4-28 kVrms) for a several frequencies $\left(10^{0}-10^{3} \mathrm{~Hz}\right)$ with $L=10 \mathrm{~cm}$; error bars are standard deviations of five measurements. Solid lines represent linear fits to the data of the form $u_{o} \propto V_{0}$; 
only data for which $V_{0} \geq 8 \mathrm{kV}$ were included in the fits to avoid effects to due to the inception voltage at $\sim 4 \mathrm{kV}$, below which no corona forms. 
(a)

(b)
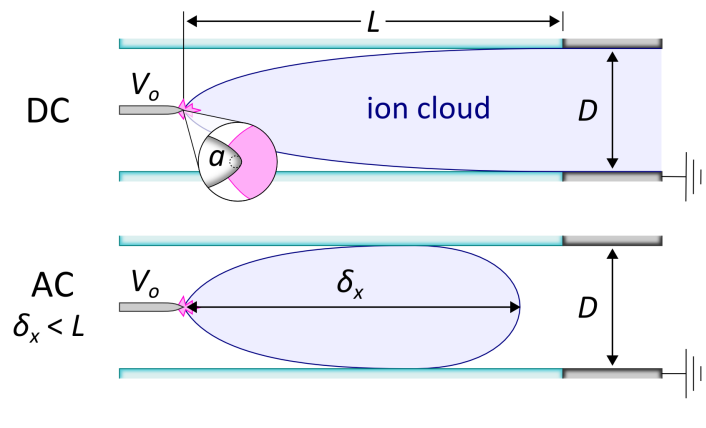

(c)

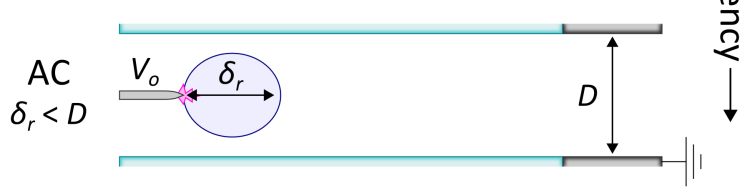

FIG. 3. Model schematic. (a) At low frequencies $\left(\omega<K V_{0} / \kappa L^{2}\right)$, the ion cloud extends a distance $L$ to the counter electrode where ions are consumed; this situation corresponds to the dc regime. (b) At high frequencies $\left(\omega>K V_{0} / \kappa L^{2}\right)$, the ion cloud extends only a distance $\delta_{x} \sim K V_{0} / \kappa \omega L$ before begin consumed by ion-ion recombination. (c) At even higher frequencies $\left(\omega>K V_{0} / \kappa D^{2}\right)$, the ion cloud is increasingly localized to within a distance $\delta_{r} \sim\left(K V_{0} / \kappa \omega\right)^{1 / 2}$ near the tip of the point electrode. 

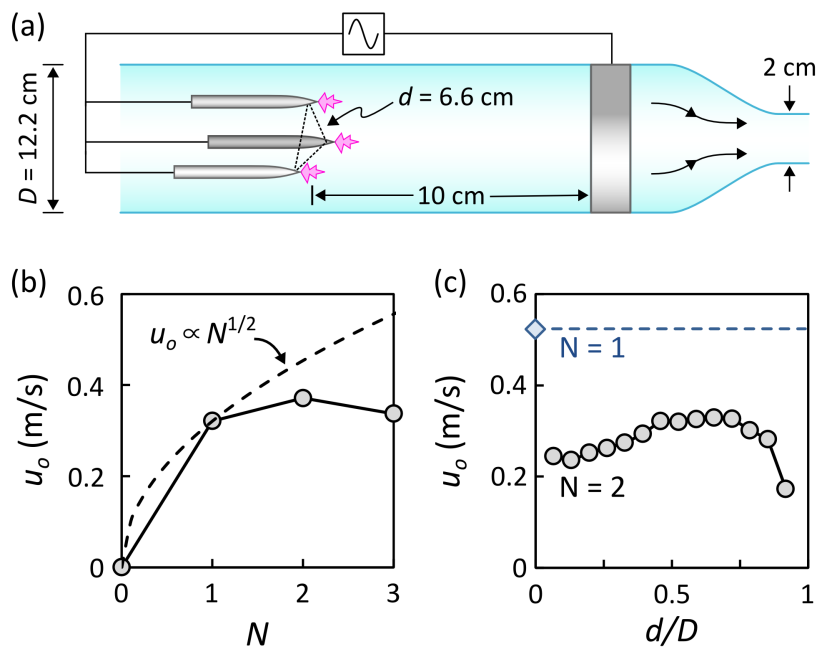

FIG. 4. Multiple parallel electrodes. (a) Experimental setup for the study of multiple electrodes. (b) Electric wind velocity $u_{o}$ as a function of the number $N$ of active corona $\left(V_{0}=10 \mathrm{kV}\right.$, $v=1 \mathrm{kHz}$ ); the dashed line shows predicted scaling $u_{o} \propto N^{1 / 2}$ assuming each corona contributes independently to the total electric force. (c) The electric wind velocity $\left(V_{0}=10 \mathrm{kV}, v=1.8 \mathrm{kHz}\right)$ for two electrodes increased with increasing separation $d$ until the electrodes neared the tube wall. The enhancement in the wind velocity with increasing separation is electric - not hydrodynamic - in origin as evidenced by analogous experiments using multiple nitrogen jets (see Supporting Information, Section 3 for details). Despite this enhancement, the wind due to two corona discharges was always less than that of a single corona positioned in the center of the tube (dashed line). 